\title{
Synchronized Response to Grayscale Image Inputs in Chaotic Cellular Neural Network
}

\author{
Masayuki Fujiwara ${ }^{\dagger}$, Akihiro Yamaguchi * \\ Department of Information and Systems Engineering, Fukuoka Institute of Technology, \\ 3-30-1 Wajiro-higashi, Higashi-ku, Fukuoka, 811-0116, JAPAN \\ Masao Kubo \\ Department of Computer Science, National Defense Academy of Japan, \\ 1-10-20 Hashirimizu, Yokosuka, Kanagawa, 239-8686, JAPAN \\ E-mail:*aki@fit.ac.jp
}

\begin{abstract}
In this study, the synchronized response in the chaotic cellular neural network for a grayscale visual stimulus was investigated in the viewpoint of neural coding. Simple gradation patterns were used as visual stimuli and the synchronized response was analyzed by the correlation of the spike firing times. As a result, synchronized responses were observed for the neurons that had similar input values and formed chaotic cell assemblies. Each assembly was distinguished from the others in terms of cross-correlation.
\end{abstract}

Keywords: chaotic synchronization, neural coding, spike response model, visual segmentation

\section{Introduction}

Recently, several information coding schemes using synchronized firing of neurons have been proposed ${ }^{1,2}$. Among them, the correlated firing is also regarded as having an important role for the information processing in the brain as a binding mechanism of neural information ${ }^{1}$. On the other hand, chaotic systems are well-known to show various complex behaviors produced by simple equations. When we consider representing the binding information by the correlated firing, the chaotic firing pattern has an advantage in its variety compared to the periodic firing pattern. Possibly, more diverse information can be represented by adopting the chaotic spike sequence compared to the periodic sequence ${ }^{3,4}$.

Authors have studied the formation of the chaotic cell assembly in the chaotic cellular neural network (Chaotic-CNN), which is a two dimensional coupled network of the chaotic spike response model (Chaotic$\mathrm{SRM})^{5,6}$. The Chaotic-SRM is an extended spike response $\operatorname{model}^{7}$ that exhibits a chaotic inter-spike interval by adding the background sinusoidal oscillation $^{4,8}$. One fundamental goal of this study is to achieve the visual segmentation using the chaotic synchronization.

In our previous study, chaotic cell assemblies were formed in a Chaotic-CNN for each localized stimulus when the stimulus was a two-dimensional binary pattern $^{6}$. For the segmentation of the real image, however, it is necessary analyzing the case in which the stimulus has analog continuous values. In this study, synchronized chaotic responses of Chaotic-CNN to the grayscale visual stimulus are numerically analyzed.

\section{Chaotic-SRM}

The spike response model (SRM) was introduced by Gerstner and Kistler ${ }^{7}$. In the SRM, the dynamics of the neuron is directly described by the kernel functions, and it is not necessary to solve the differential equations for the simulation. We proposed the Chaotic-SRM, which is

\footnotetext{
${ }^{\dagger}$ Present affiliation is Japan Advanced Institute of Science and Technology.
} 
an extended SRM, to show the chaotic inter-spike intervals ${ }^{5}$. The definition of Chaotic-SRM is as follows. The membrane potential $u(t)$ of the neuron at the time $t$ is defined as

$$
u(t)=u_{\text {rest }}+\eta\left(t-t^{*}\right)+\beta,
$$

where $u_{\text {rest }}, t^{*}$, and $\beta$ denote the resting potential of the neuron, the last firing time of this neuron, and the external input, respectively; the kernel function $\eta$ describes the response of the membrane potential after firing. The definition of the kernel function $\eta$ is

$$
\eta\left(t-t^{*}\right)=-\eta_{\text {init }} \exp \left(\frac{t-t^{*}}{\tau_{\eta_{0}}}\right) \Theta\left(t-t^{*}\right),
$$

where $\tau_{\eta_{0}}$ is the time constant of the spike response and $\Theta$ is a step function such that $\Theta(s)$ is 1 for $s \geq 0$ and 0 for the other values. In this model, when the membrane potential exceeds the threshold value $\theta$, the neuron is firing and the membrane potential is reset by the update of the last firing time $t^{*}$. The term $-\eta_{\text {init }}$ is an initial value of the kernel function $\eta$ after firing. In the original SRM, this term is constant. Therefore, the original SRM is periodically firing, and its period is determined by the external input value $\beta$.

We extended the original SRM to show the chaotic response by adding a background sinusoidal oscillation in the same way as the bifurcating neuron ${ }^{8}$ and the chaotic pulse coupled neural network ${ }^{4}$. In the extended SRM, the background oscillation is added to the term $\eta_{\text {init }}$ :

$$
\eta_{\text {init }}=\eta_{0}-A_{\eta_{0}} \sin \left(2 \pi \omega_{\eta_{0}} t^{*}\right)
$$

where $\eta_{0}$ denotes the constant $\eta_{\text {init }}$ in the original model, and $A_{\eta_{0}}$ and $\omega_{\eta_{0}}$ denote the amplitude and the frequency of the background oscillation, respectively. In this article, we call this extended model the "ChaoticSRM". The Chaotic-SRM exhibits various chaotic behaviors depending on the parameter values of $A_{\eta_{0}}$ and the external input $\beta$.

The bifurcation diagram and the Lyapunov exponent for the external input $\beta$ are obtained by numerical simulation ${ }^{6}$ (Fig. 1); the parameter values of the Chaotic-SRM are set as follows: $u_{\text {rest }}=-70 \mathrm{mv}$, $\theta=-35 \mathrm{mv}, \eta_{0}=55, \tau_{\eta_{0}}=10 \mathrm{~ms}, \omega_{\eta_{0}}=0.75 / 2 \pi$, and $A_{\eta_{0}}=10.9$. In the bifurcation diagram (Fig. 1(a)), the period doubling bifurcation, which is a typical behavior of the chaotic system, was observed. In Fig. 1(b), several regions where the Lyapunov exponent are positive are shown; these regions correspond to the chaotic region in the bifurcation diagram.

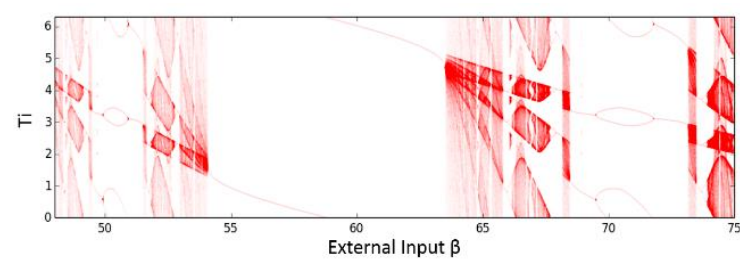

(a)

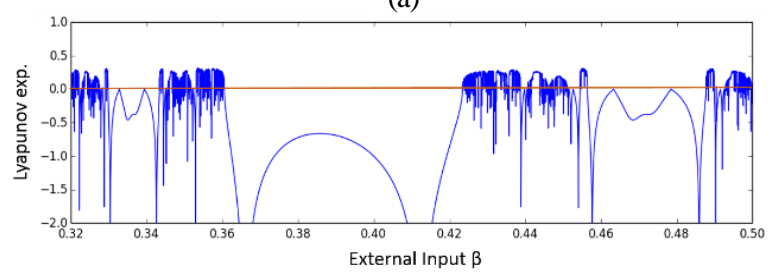

(b)

Fig.1. (a) Bifurcating diagram and (b) Lyapunov Exponent ${ }^{6}$.

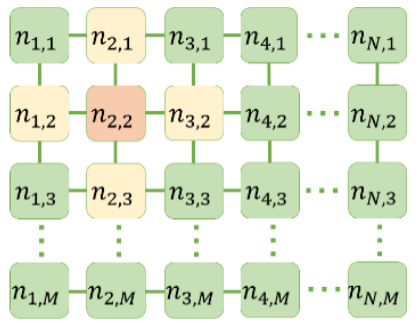

Fig. 2. Chaotic-CNN ${ }^{6}$.

\section{Chaotic-CNN}

The Chaotic-CNN is defined as a two dimensional coupled system of the Chaotic-SRM ${ }^{6}$. In the Chaotic$\mathrm{CNN}$, each neuron is placed in the $N \times M$ lattice and connected to the nearest neighbors in four directions (up, down, left, and right), as shown in Fig. 2. Let $n_{x, y}$ be a neuron located at the position $(x, y)$. The membrane potential of $n_{x, y}$ is denoted by $u_{x, y}$, and it is defined as

$$
\begin{gathered}
u_{x, y}(t)=u_{\text {rest }}+\eta\left(t-t_{x, y}^{*}\right)+\beta_{x, y} \\
+\xi \times\left[o_{x-1, y}(t)+o_{x+1, y}(t)+o_{x, y-1}(t)\right. \\
\left.+o_{x, y+1}(t)\right]
\end{gathered}
$$

where $t_{x, y}^{*}, \beta_{x, y}$, and $\xi$ denote the last firing time, the external input of $n_{x, y}$, and the coupling weight, respectively. The boundary condition is fixed as follows: $o_{0, y}(t)=o_{N+1, y}(t)=o_{x, 0}(t)=o_{x, M+1}(t)=0$.

The function $o_{x^{\prime}, y^{\prime}}$ is the output from the connected neuron $n_{x^{\prime}, y^{\prime}}$ and it is also defined as

$$
o_{x^{\prime}, y^{\prime}}(t)=\sum_{t_{x, y}^{*}<t_{x^{\prime}, y^{\prime}}^{(k)}<t} \varepsilon\left(t-t_{x^{\prime}, y^{\prime}}^{(k)}\right),
$$

where $t_{x^{\prime}, y^{\prime}}^{(k)}$ denotes the $k$-th firing time of the neuron $n_{x^{\prime}, y^{\prime}}$. The kernel function $\varepsilon$ describes the response of 
the synaptic connection. The definition of the kernel function $\varepsilon$ is

$$
\varepsilon(s)=\frac{s}{\tau_{\varepsilon}} \exp \left(-\frac{s}{\tau_{\varepsilon}}\right) \Theta(s),
$$

where $\tau_{\varepsilon}$ is the time constant of the synaptic connection.

\section{Correlation Analysis}

In this article, the synchronized response is analyzed by examining the correlation. As an index of the synchronized response, the cross-correlation between two neurons is analyzed. Let $S_{x, y}$ be a set of the firing times of the neuron $n_{x, y}$. The cross-correlation between $S_{x, y}$ and $S_{x^{\prime}, y^{\prime}}$ with time shift $\phi$ is defined as

$$
\begin{aligned}
& C C\left(S_{x, y}, S_{x^{\prime}, y^{\prime}} ; \phi\right)= \\
& \frac{\#\left(\left\{t_{x, y}^{(k)} \in S_{x, y}\left|\exists t_{x^{\prime}, y^{\prime}}^{(l)} \in S_{x^{\prime}, y^{\prime}},\right| t_{x, y}^{(k)}-t_{x^{\prime}, y^{\prime}}^{(l)}-\phi \mid \leq \Delta s\right\}\right)}{\#\left(S_{x, y}\right)},
\end{aligned}
$$

where $\#(X)$ denotes the number of elements of $X$, and $\Delta s$ is the time resolution of the coincident firing. In this work, $\Delta s$ is set to $0.5 \mathrm{~ms}$. The auto-correlation is also defined as $A C\left(S_{x, y} ; \phi\right)=C C\left(S_{x, y}, S_{x, y} ; \phi\right)$. The maximal value of the cross-correlation is defined as

$$
C C^{*}\left(S_{x, y}, S_{x^{\prime}, y^{\prime}}\right)=\max _{\phi} C C\left(S_{x, y}, S_{x^{\prime}, y^{\prime}} ; \phi\right) .
$$

In the case of $C C^{*}=C C\left(S_{x, y}, S_{x^{\prime}, y^{\prime}} ; \phi\right) \simeq 1, S_{x, y}$ and $S_{x^{\prime}, y^{\prime}}$ are synchronized with the time shift $\phi$. In the case of $A C^{*}=A C\left(S_{x, y} ; \phi \neq 0\right) \simeq 1, S_{x, y}$ is periodic with period $\phi$. When the spike sequence $S_{x, y}$ is chaotic, $A C\left(S_{x, y} ; \phi\right)$ exponentially decays for the time shift $\phi$.

\section{Numerical Experiments}

As numerical experiments, we simulated the Chaotic$\mathrm{CNN}$ for $20 \times 20$ grayscale image patterns, shown in Fig. 3(a) and Fig. 4(a), where the parameters of the single neuron are set at the same values mentioned in section 2, and the parameters of the coupling are set as follows: $\tau_{\varepsilon}=0.5 \mathrm{~ms}$ and $\xi=4$. The grayscale pixel value $g_{x, y} \in\{0,1, \cdots, 255\}$ is mapped to the external input $\beta_{x, y} \in\left[\beta_{0}, \beta_{1}\right]$ such that

$$
\beta_{x, y}=\left(\beta_{1}-\beta_{0}\right) \times \frac{g_{x, y}}{255}+\beta_{0} .
$$

In this simulation, we chose the interval $[48,54]$ as $\left[\beta_{0}, \beta_{1}\right]$, which includes the chaotic region in the bifurcation diagram (Fig. 1).

The input pattern shown in Fig. 3(a) is a simple gradation pattern from black to white in the direction of the $\mathrm{x}$-axis. The neurons aligned in the direction of the $\mathrm{y}$ axis have the same input value. A response of the Chaotic-CNN is shown in Fig. 3(b) as a raster plot of firing times, where the abscissa represents the time and

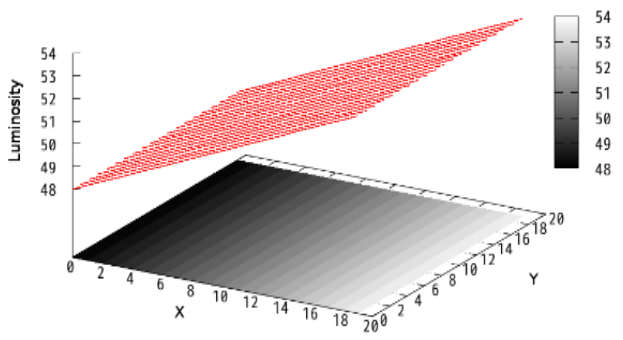

(a) External input $\beta_{x, y}$ as a visual stimulus.

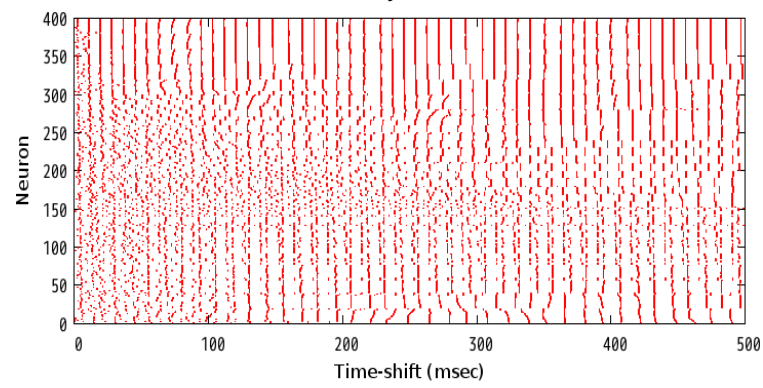

(b) Raster plot of the synchronized response

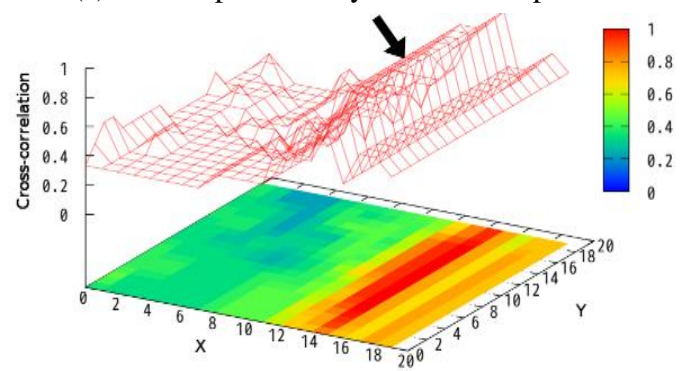

(c) Maximal cross-correlation between the neuron indicated by the arrow and the other neurons.

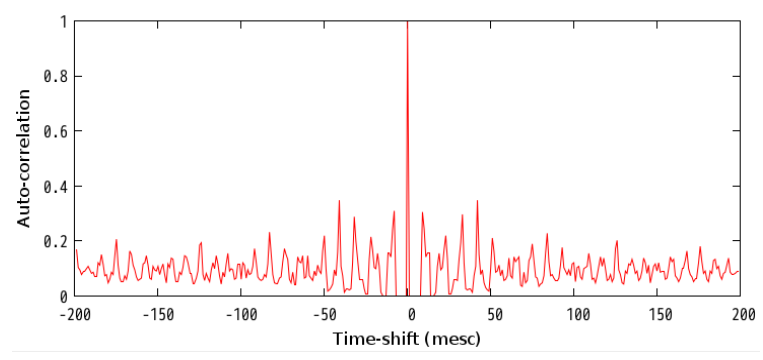

(d) Auto-correlation profile of the neuron indicated in (c).

Fig. 3. Response to the simple gradation pattern.

the ordinate represents an identifier of the neuron such that $i d=20 x+y$ for the neuron $n_{x, y}$. Spike responses are roughly synchronized for each input value. The maximal cross-correlations between the neuron indicated by the arrow and the other neurons were calculated (Fig. 3(c)). As shown in Fig. 3(c), high correlation is observed for the neurons in the direction of the y-axis, whereas a relatively lower correlation is observed for the others. The auto-correlation profile was 


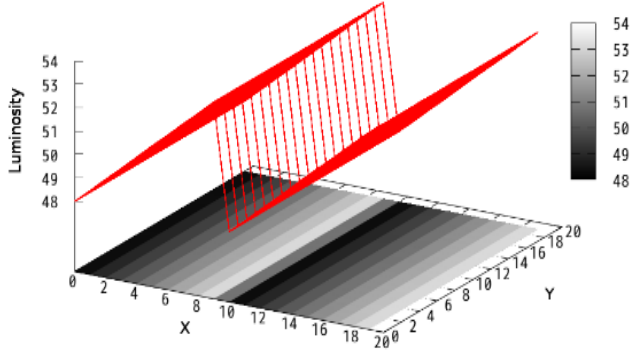

(a) External input $\beta_{x, y}$ as a visual stimulus.

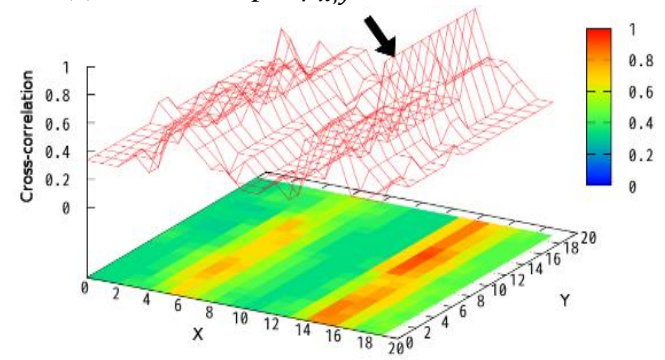

(b) Maximal cross-correlation for the chaotic response.

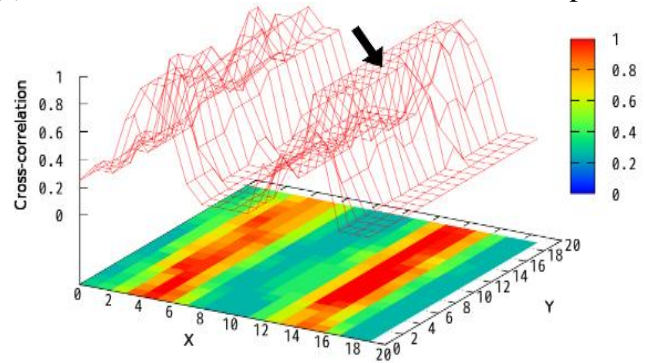

(c) Maximal cross-correlation for the periodic response.

Fig. 4. Response to the repeated gradation pattern.

also calculated for the neuron indicated in Fig. 3(c). As shown in Fig. 3(d), an exponential decay of the autocorrelation is observed. These results indicate the formation of the chaotic cell assemblies.

To highlight the differences between the chaotic and the periodic cell assemblies, the responses to a twice repeated gradation pattern, shown in Fig. 4(a), were analyzed. The maximal cross-correlations for the case of the chaotic and periodic spike responses are shown in Fig. 4(b) and (c), respectively. For the periodic spike responses, the amplitude of the background oscillation $A_{\eta_{0}}$ was set to 0 to generate periodic spike responses. In this case, the neuron indicated by the arrow exhibits high correlation with the neurons in the two regions where the neurons have a similar input value (Fig. 4(c)). In terms of correlation, these two regions are not distinguished from each other. On the other hand, in the case of the chaotic spike response, the neuron indicated by the arrow has a high correlation with the neurons in one region, included itself, and has a relatively low correlation with the other neurons. In this case, the chaotic cell assemblies invoked by the grayscale input are distinguished from the others even if they have a similar input value.

\section{Conclusions}

For the grayscale visual stimulus, the synchronized response of the Chaotic-CNN was analyzed by the correlation of the spike firing times. As a result, synchronized responses to the similar input value and a formation of chaotic cell assemblies were observed. These results indicate the possibility to achieve visual segmentation using the synchronized chaotic response. The analysis of the real image input will be performed in our future works.

\section{Acknowledgements}

This work was partially supported by JSPS KAKENHI Grant Numbers 24650120.

\section{References}

1. Gray, C.M., Koenig, P., Engel, A. K., and Singer, W., Oscillatory responses in cat visual cortex exhibit intercolumnar synchronization which reflects global stimulus properties, Nature, 338, pp.334-337, 1989.

2. Fuji, H., Ito, H., Aihara, K., Ichinose, N., and Tsukada, M., Dynamical cell assembly hypothesis - Theoretical possibility of spatio-temporal coding in the cortex, Neural Networks, Vol.9, No.8, pp.1303-1350, 1996.

3. Yamaguchi, A., Okumura, N., Chaki, H., and Wada, M., Chaotic synchronized cluster in the network of spike response neurons, IEICE Tech. Rep., Vol.99, No.685, NC99-119, pp. 15-20, Mar. 2000 (in Japanese).

4. Yamaguti, Y., Ishimura, K., and Wada M., Chaotic synchronized assembly in Pulse Coupled Neural Networks, IEICE Tech. Rep., Vol.101, No.615, NC200198, pp.127-134, Jan. 2002 (in Japanese).

5. Yamaguchi, A., On a chaotic synchronization of one-way coupled two spike response neurons, Fukuoka Institute of Technology Reports of Computer Science Laboratory, Vol.24, pp.1-6, 2013 (in Japanese).

6. Yamaguchi, A., On an information coding using localized synchronization in the two dimensional coupled system of chaotic spike response neurons, Fukuoka Institute of Technology Reports of Computer Science Laboratory, Vol.25, pp.1-6, 2014 (in Japanese).

7. Gerstner, W., and Kistler, W., Spiking Neuron Models: Single Neurons Populations Plasticity, Cambridge University Press, 2002.

8. Lee G., and Farhat, N.H., The Bifurcating Neuron Network 1, Neural Networks, Vol. 14, pp.115-131, 2001. 